1 Sarkissian, I. V., Physiol. Plant, 19, 328 (1966).

2 Mitchell, K. K., and Sarkissian, I. V., J. Fxp. Botany, 17, 838 (1960).

sarkissian, I. V., Biochemistry and Physiology of Plant Grouth Substances (edit. by F. Wightman and G. Setterfield), 473 (The Runge Press,

4 Zelik, M. H., and Nissl, D., Naturuissenschaften, 55, 84 (1968).

5 Gubinsky, J. S., and Cleland, W. W., Biochemistry, " 566 (1968).

' Mr.yind, J., Wyman, J., and changeux, J. P., J. Mol. Biol., 12, 88 (1965).

\section{Evolutionary Rates and the Inference of Evolutionary Tree Forms}

SEveral methods have been developed recently to decide which of a set of alternative trees is the most consistent with genetic, biochemical or morphological information about populations of present day organisms ${ }^{1-5}$. Most of the methods involve a "minimum evolution" hypothesis to the effect that the observed differences have been established by the smallest possible number of mutations or unit biochemical or morphological changes. The uubious nature of the minimum evolution hypothesis has been emphasized by Inger ${ }^{6}$ and Rogers et al. ${ }^{7}$, and the difficulties involved in selecting strictly comparable units of morphological change have been discussed by Lerman ${ }^{8}$. The fact that the number of possible distinct tree forms increases very rapidly with increase in the number of populations leads to computational difficulties; several authors have used some form of average-linkage eluster analysis on dissimilarity or association measures between populations to obtain a preliminary reduction in the number of tree forms ${ }^{2,3,9}$

A subset of a set of populations can be said to be monophyletic if its component populations have diverged from one another more recently than from any other population. The method proposed here finds subsets of a set of populations which can be assumed to be monophyletic given a particular assumption about evolutionary rates. It thus provisionally divides the set of possible tree forms into an acceptable group and an unacceptablo group.

The hicrarchic structure of evolutionary trees for genetically isolated populations ensures that the times sine divergence obey the ultrametric inequality. That is, the time since divergence of any pair of populations $a$ and $b$ is less than or equal to the larger of the times since divergence of $a$ or $b$ from any other population. If evolutionary rates had been constant, then the pairwise dis similaritics of present day populations would be monotone with the times since their divergence, and would therefore be viltrametric. The correct evolutionary tree form could then bo obtained immediately from the rolative dis-

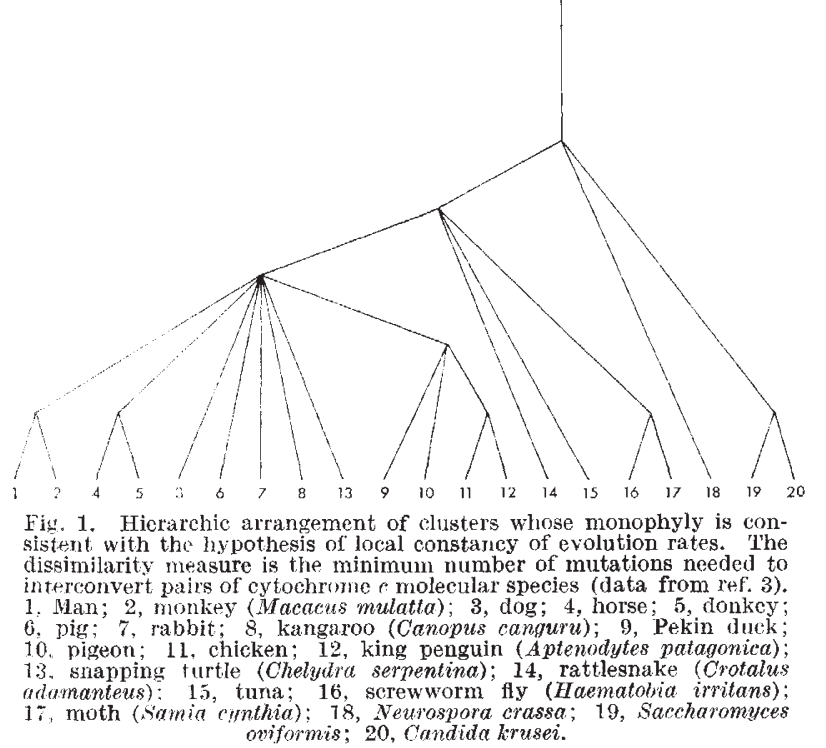

similarities. It is well known that for genetically isolated populations from a wide range of groups of organisms the dissimilarities based on morphological and biochemical information rarely have ultrametric structure. The extent to which evolutionary rates have been inconstant with respect to a given measure of dissimilarity is roughly indicated by measures of the extent to which present day dissimilarities depart from ultrametric structure (compare refs. 10 and 11).

A much weaker altemative to the generally false assump. tion of constancy of evolutionary rates is the assumption that evolutionary rates have usually ben locally constant. That is

$$
d(a, b)<d(b, c) \longleftrightarrow-\rightarrow t(a, b)<t(b, c)
$$

where $d$ is the present day dissimilarity and $t$ is the tim. since divergence of a pair of populations. All subsets of a set of populations which can be assumed to be monophyletic without violating this condition can be obtained from the rank ordering of pairwise dissimilarities by finding all ball-clusters. A ball-cluster is a subset of the set of populations for which each component population is less dissimilar from each other population in the subset than it is from any population not in the subset. An algorithm for finding all ball-clusters determined by the rank ordering of pairwise dissimilarity measures on a set of objects has been programmed in Fortran by C. J. van Rijsbergen and will be described in a later publication.

An example of the application of the method using bicchemical data from Fitch and Margoliash ${ }^{3}$ is shown in Fig. 1. In this case the monophyly of the ball-clusters is consistent with what can bo inferred about the phylogenet ic branching sequence on independent palaeontological and taxonomic grounds. The mothod has been checked also on various recently published biochemical, serological and morphological data and has in each case yielded results which are consistent with what can be inferred about phylogeny on independent grounds.

Two tentative conclusions can be drawn from these results. First, in making inferences about phylogeny from information about contemporary organisms it may be more reasonable to attempt to exclude those tree forms which are inconsistent with the data given particular hypotheses about evolutionary rates than to attempt to infer a unique tree form from the data. Second, it seems that although evolutionary rates measured in various ways are not constant they aro often locally constant. This might be explained either by supposing evolutionary rates to be partly under direct genetic control or by supposing that predominant among the selection pressurcs which determine evolutionary rates are the internal selec. tion pressures imposed by the chemical environment. In either case the more similar the genomes of populations the more similar would be their rates of evolutionary divergence.

This work was carried out during the tenure of a Roya! Society scientific information research fellowship and an SRC research grant by N. J.

$$
\begin{aligned}
& \text { N. JaRdine } \\
& \text { C. J. VAN RiJSHERGEN } \\
& \text { C. J. JARDTNE }
\end{aligned}
$$

King's College Research Contre,

University of Cambridge.

Reccived July 24, 1969.

${ }^{1}$ Camin, J. H., and Sokal, R. R., Evolution, 19, 311 (1965).

"Cavalli-Sforza, L. L., and Edwards, A. W. F., Evolution, 21, $550(1967$

${ }^{3}$ Fitch, W. M., and Margoliash, E., Science, 155, $279(1967)$.

${ }^{4}$ Kluge, A. G., and Tarris, J. S., Syst. Zool., 18, 1 (1969).

${ }^{5}$ Wagner, W. H., in Recent Advances in Botany, 841 (Cniversity of Toronto Press, 1961).

' Inger, R. F., Evolution, 21, 369 (1967).

? liogers, D. J., Fleming, H. S., and Estabrook, G., in Firilutionary Biolisg. (edit. by Dobzhansky, T., Hecht, M. K., and Steerr. W. (.). 1, 16 (Appleton-Century-Crofts, New York, $1 9 6 \longdiv { \imath }$ ).

${ }^{8}$ Lerman, A., Evolution, 19, 16 (1965).

'Taylor, R. J., and Campbell, D., Evolution, 23, 153 (1969).

${ }^{10}$ Jardine, N., and Sibson, R., Comp. J., 11, 177 (1968).

${ }_{11}^{11}$ Jardine, N., Syst. Zool., 18, 37 (1969). 